\title{
Perfil de formação e produção científica do fisioterapeuta pesquisador no Brasil
}

\author{
Profle of scientific academic background and production of researcher physical therapists in Brazil \\ Perfil formativo y producción cientifica del fisioterapeuta investigador en Brasil
}

\begin{abstract}
Natália Guimarães Melo', Isabela Alves Cunha², Júlia Ferreira Alves ${ }^{3}$, Amanda Lindolpho Santos ${ }^{4}$, Anna Paula Nogueira ${ }^{5}$, Beatriz Correa Lima ${ }^{6}$, Bruna Viani Dias ${ }^{7}$, Jeovana Souza Cardoso ${ }^{8}$, Jhennyfer Gonzaga de Oliveira Rocha ${ }^{9}$, Nathálya Pereira Portugal ${ }^{10}$, Rayssa Gabrielly de Araujo", Rayssa Martins de Souza ${ }^{12}$, Roberta Larissa Oliveira Paulino ${ }^{13}$, Tânia Cristina Dias da Silva Hamu'14, Cibelle Kayenne Martins Roberto Formiga ${ }^{15}$
\end{abstract}

\begin{abstract}
RESUMO I O objetivo deste estudo foi avaliar o perfil de formação e produção científica do fisioterapeuta pesquisador brasileiro. Trata-se de um estudo transversal, realizado por meio de levantamento dos currículos profissionais cadastrados na Plataforma Lattes do Conselho Nacional de Desenvolvimento Científico e Tecnológico (CNPq), atualizados nos últimos 5 anos. As principais variáveis analisadas foram sexo, distribuição dos fisioterapeutas pelo Brasil, tipo de instituição de graduação, local de atuação, formação complementar, produções científicas e participação em eventos. Foram encontrados 47.741 currículos, dos quais 17.864 estavam dentro dos critérios de seleção da pesquisa e foram analisados. Predominaram fisioterapeutas do sexo feminino (75,5\%) e da região Sudeste (35\%). A maioria é graduada em instituições privadas (75\%), realizou pósgraduação lato sensu (61,4\%) e trabalhou em universidades (30\%). Existem diferenças entre os sexos com relação às produções, os eventos e a formação complementar, com o melhor desempenho do sexo masculino ( $p<0,001)$ - os graduados em instituição pública têm mais produções,
\end{abstract}

eventos e atividades de formação complementar $(p<0,001)$. Os profissionais graduados em instituições privadas, porém, realizam mais cursos de pós-graduação lato sensu ( $\mathrm{p}=0,05)$. Conclui-se, portanto, que a maioria dos fisioterapeutas pesquisadores do Brasil é do sexo feminino, graduada na região Sudeste, formada em instituições privadas, realizou pós-graduação lato sensu e trabalha em universidades. Apesar da maioria feminina, os profissionais do sexo masculino e os formados em instituições públicas apresentam maior quantidade de produções, participações em eventos e formações complementares.

Descritores | Fisioterapia; Indicadores de Produção Científica; Currículo; Pesquisadores.

ABSTRACT| To evaluate the academic background and scientific productions' profile of researcher physiotherapists in Brazil. The study was cross-sectional in design and performed by analyzing the professional resumes that have been updated within the last 5 years in the Lattes Platform of the Conselho Nacional de Desenvolvimento

\footnotetext{
Universidade Estadual de Goiás (UEG), Goiânia (GO), Brasil. E-mail: natalia-gmelo@hotmail.com. Orcid: 0000-0001-6971-3103 2Universidade Estadual de Goiás (UEG), Goiânia (GO), Brasil. E-mail: isaalvesfisio@gmail.com. Orcid: 0000-0002-7183-2192 3Universidade Estadual de Goiás (UEG), Goiânia (GO), Brasil. E-mail: juliafalves01@gmail.com. Orcid: 0000-0002-9108-6596 ${ }^{4}$ Universidade Estadual de Goiás (UEG), Goiânia (GO), Brasil. E-mail: lindolphoamanda@gmail.com. Orcid: 0000-0001-7724-1943 5Universidade Estadual de Goiás (UEG), Goiânia (GO), Brasil. E-mail: annanogueeira@gmail.com. Orcid: 0000-0001-5605-3863 6Universidade Estadual de Goiás (UEG), Goiânia (GO), Brasil. E-mail: beatrizclima03@hotmail.com. Orcid: 0000-0003-4902-5287 7Universidade Estadual de Goiás (UEG), Goiânia (GO), Brasil. E-mail: brunavianidias@gmail.com. Orcid: 0000-0002-0053-9757 8Universidade Estadual de Goiás (UEG), Goiânia (GO), Brasil. E-mail: jeovana.souza@hotmail.com. Orcid: 0000-0001-6929-1350 'Universidade Estadual de Goiás (UEG), Goiânia (GO), Brasil. E-mail: jhennyferfisio@gmail.com. Orcid: 0000-0003-1726-4478 10 Universidade Estadual de Goiás (UEG), Goiânia (GO), Brasil. E-mail: nathalya.pportugal@gmail.com. Orcid: 0000-0001-7801-6035 "Universidade Estadual de Goiás (UEG), Goiânia (GO), Brasil. E-mail: rayssgabrielly25@gmail.com. Orcid: 0000-0002-3083-7173 12Universidade Estadual de Goiás (UEG), Goiânia (GO), Brasil. E-mail: rayssa.msouza@outlook.com. Orcid: 0000-0002-7680-7332 ${ }^{13}$ Universidade Estadual de Goiás (UEG), Goiânia (GO), Brasil. E-mail: robertapaulinoo@gmail.com. Orcid: 0000-0002-5485-6010 ${ }^{14}$ Universidade Estadual de Goiás (UEG), Goiânia (GO), Brasil. E-mail: tania.ft@gmail.com. Orcid: 0000-0002-8776-5991

15Universidade Estadual de Goiás (UEG), Goiânia (GO), Brasil. E-mail: cibellekayenne@gmail.com. Orcid: 0000-0002-5837-297X
} 
Científico e Tecnológico (CNPq). The main variables analyzed were sex, geographical distribution, kind of undergraduate institution, working location, complementary training, scientific production, and participation in events. The analysis was carried out on 17,864 curricula which were within the selection criteria, out of a total of 47,741 . There was a predominance of female therapists (75.5\%) and therapists from the southeast region (35\%). Most have completed their undergraduate degree in private institutions (75\%) and graduate programs (61.4\%), and work at universities (30\%). Males perform better ( $p<0.001$ ) in scientific productions, participation in events, and complementary training activities. Graduates from public institutions have more scientific productions, events, and complementary training activities $(p<0.001)$. However, professionals that have completed their undergraduate degree in private institutions do graduate programs the most $(p=0.05)$. Most researcher physical therapists in Brazil are females, have completed their undergraduate degrees in the Southeast region and in private institutions, have completed graduate programs and work in universities. Despite the female predominance, male professionals and those therapists who have completed their undergraduate degree from public institutions have a greater amount of scientific productions, participation in events and complementary degrees.

Keywords | Physical therapy; Scientific Production Indicators; Curriculum; Researchers.

RESUMEN | El objetivo de este estudio fue analizar el perfil de formación y de producción científica del fisioterapeuta investigador brasileño. Este es un estudio transversal, realizado a partir de búsqueda de currículums profesionales registrados en la Plataforma Lattes del Consejo Nacional de Desarrollo Científico y Tecnológico (CNPq), actualizados en los últimos 5 años. Las principales variables analizadas fueron: género, distribución de fisioterapeutas por Brasil, tipo de institución de grado, lugar de actuación, formación complementaria, producción científica y participación en eventos. Se encontraron 47.741 currículums, de los cuales 17.864 cumplían los criterios de selección del estudio para el análisis. Hubo una mayor prevalencia de fisioterapeutas mujeres (75,5\%) y de la región Sudeste (35\%). La mayoría son egresadas de instituciones privadas (75\%), completaron los estudios de posgrado lato sensu $(61,4 \%)$ y trabajaron en universidades (30\%). Existen diferencias entre géneros en cuanto a producciones, eventos y formación complementaria, con el mejor desempeño para los varones ( $p<0,001$ ), los egresados de instituciones públicas cuentan con más producciones, eventos y actividades de formación complementaria $(p<0,001)$. Los profesionales graduados de instituciones privadas, sin embargo, hacen más cursos de posgrado lato sensu $(p=0,05)$. Se concluye que la mayoría de los profesionales de fisioterapia investigadores en Brasil son mujeres, egresadas en la región Sudeste, con formación en instituciones privadas, con estudios de posgrado lato sensu y que trabajan en universidades. A pesar de la mayoría femenina, los profesionales masculinos y aquellos que estudiaron en instituciones públicas son los que tienen mayor número de producciones, participaciones en eventos y formaciones complementarias.

Palabras clave I Fisioterapia; Indicadores de Producción Científica; Currículum; Investigadores.

\section{INTRODUÇÃO}

A fisioterapia é mundialmente reconhecida como uma profissão com atribuições diferenciadas e complementares às da área de saúde como medicina, educação física e enfermagem. A prática começou a se constituir como profissão no século $\mathrm{XIX}^{1}$, quando surgem as primeiras escolas europeias voltadas para a formação desses profissionais. Na América do Norte e na Oceania, escolas de semelhante formação surgem no início do século $\mathrm{XX}^{2}$.

Ocorreu um evidente crescimento da profissão, visto que a denominação do profissional sofreu alterações ao longo da história com a substituição de termos como "massagistas" por "técnicos" e de "subordinados da medicina" para profissionais com autonomia, poder de diagnóstico e atuação direta no tratamento do paciente ${ }^{3,4}$.
Em Portugal, a ascensão da fisioterapia se deu diante das necessidades impostas pela II Guerra Mundial, sendo necessária a formação especializada de um grupo de profissionais que soubesse aplicar recursos individualizados e eficazes para a reabilitação de excombatentes. O reconhecimento da profissão no país ainda não foi instaurado por completo, mas avançou em passos largos nas últimas três décadas ${ }^{2}$. No Brasil, seu avanço teve como marco inicial a Lei no 938, de 13 de outubro de $1969^{5}$, sendo reconhecida como profissão de nível superior que atua diretamente na promoção, prevenção e recuperação da saúde ${ }^{6}$.

Nos Estados Unidos, é estimado um crescimento desproporcional de fisioterapeutas entre os anos de 2013 e 2020, começando como um período de escassez seguido por um aumento do número de profissionais 
em formação ${ }^{7}$. No Brasil, entre os anos de 1995 e 2015, houve um aumento expressivo no número de cursos de fisioterapia observado principalmente em instituições de ensino privado, principalmente na região Sudeste ${ }^{8}$. Consequentemente, houve o crescimento da capacitação e da produção científica, demonstrando ascensão no conhecimento científico e pesquisas no Brasil ${ }^{9}$.

Entre os indicadores de crescimento quantitativo da profissão, observou-se percentualmente maior quantidade de indivíduos do sexo masculino, o que perdurou por vários anos. $\mathrm{O}$ processo de feminilização da área da saúde, contudo, tem combatido estigmas vinculados à contextos históricos e socioculturais, transformando esse cenário predominantemente masculino ao longo dos últimos tempos em diversos países ${ }^{10,11}$.

No âmbito da formação profissional, a instituição de ensino superior em que se formam os profissionais fisioterapeutas é um fator importante a ser considerado. A ampliação da oferta de cursos de fisioterapia impactou a qualidade do processo de formação profissional. Nas avaliações do Exame Nacional de Desempenho dos Estudantes (Enade) e no Conceito Preliminar de Curso realizados pelo Ministério da Educação (MEC), as instituições de ensino público ainda possuem um melhor desempenho - mesmo com a evolução conceitual obtida por instituições privadas. A proporção de resultados insuficientes (ou que apenas alcançam o critério mínimo) é preocupante, pois coloca em xeque a qualidade da formação do bacharel em fisioterapia no Brasil ${ }^{12}$.

Há, no Brasil, 210 milhões de habitantes ${ }^{13}$ e, desse total, em torno de 240 mil são fisioterapeutas ${ }^{14}$ - o que representa 1,14 fisioterapeutas para cada 1.000 habitantes. É um número expressivo de profissionais ativos no mercado de trabalho que atuam em todos os níveis de atenção em saúde e em todas as faixas etárias da população brasileira. Não basta, contudo, apenas enumerar os fisioterapeutas no mercado de trabalho; é preciso que os profissionais estejam qualificados para realizar condutas cada vez mais respaldadas em conhecimentos científicos e baseadas em evidências ${ }^{15,16}$.

O profissional graduado em fisioterapia deve ter conhecimento teórico e prático qualificado, apresentar habilidades e competências de um cidadão crítico e socialmente comprometido, ter a capacidade de produzir conhecimento científico qualificado e de atuar nos níveis primário, secundário e terciário de atenção à saúde ${ }^{17}$. Sob esse aspecto, o avanço e o reconhecimento da fisioterapia dependem do reflexo significativo de suas evidências científicas, que atribuem credibilidade e eficácia à sua atuação e resultados positivos com suas intervenções ${ }^{18}$.

Muitos obstáculos ainda são identificados no processo de pesquisa e produção científica na área da fisioterapia. Os profissionais relatam deficits tanto na matriz curricular da graduação quanto na habilidade de pesquisa, na síntese e na avaliação crítica da literatura ${ }^{19}$. Grande parte dos fisioterapeutas pesquisadores aproxima-se mais da prática baseada em evidência apenas na pós-graduação stricto sensu, quando passa a guiar sua conduta profissional a partir do raciocínio científico que objetiva resultados significativos para a atuação clínica ${ }^{20}$. A educação e formação continuada, portanto, se fazem necessárias a fim de qualificar o profissional e ampliar suas linhas de conhecimento e habilidades ${ }^{19}$.

A fisioterapia vem buscando, gradativamente, reconhecimento da sociedade e das políticas públicas de saúde, sendo que a qualificação profissional e a participação no meio científico são aspectos importantes a serem considerados. Ainda existem diversos aspectos a serem melhorados, como, por exemplo, a quantidade de pesquisadores, a quantidade de programas de pósgraduação e a quantidade de conhecimento científico de qualidade produzido e divulgado ${ }^{21}$.

Conhecer o perfil dos profissionais fisioterapeutas brasileiros, especificamente daqueles voltados à produção de conhecimento científico (pesquisadores), é de fundamental importância para identificar lacunas na formação e deficits nas áreas de atuação e distribuição dos profissionais pelo país. Por conseguinte, o objetivo do estudo é analisar o perfil de formação e produção científica do fisioterapeuta pesquisador brasileiro.

\section{METODOLOGIA}

Este é um estudo observacional transversal, de base bibliométrica, com amostra de 17.864 currículos de fisioterapeutas pesquisadores brasileiros cadastrados na plataforma Lattes do CNPq. Participaram do estudo os profissionais que atenderam aos seguintes critérios de inclusão: apresentar currículo na plataforma Lattes, ser formado em fisioterapia por instituição pública ou privada, ambos os sexos. Os critérios de exclusão foram: não apresentar o currículo atualizado nos últimos 5 anos (de 2013 a 2018), currículos duplicados por atualização da plataforma ou com informações incompletas sobre a formação profissional do indivíduo. 
A Plataforma Lattes do CNPq (http://lattes.cnpq.br/) foi selecionada para a pesquisa pelos seguintes motivos: ser um instrumento oficial, público e gratuito para o acesso a currículos de profissionais de todo Brasil; ser um instrumento com um mínimo de garantia de veracidade de informações, já que para cadastrar e publicar os dados na plataforma é necessário atestar a veracidade dos dados lançados; ser uma plataforma de integração de bases de dados de currículos, grupos de pesquisa e de instituições em um único sistema de informação; ser um instrumento padrão nacional no registro do percurso acadêmico de estudantes e pesquisadores do Brasil; ser adotado pela maioria das instituições de fomento, universidades e institutos de pesquisa do país.

$\mathrm{O}$ acesso à plataforma foi realizado, de início, por meio do seguinte filtro de busca: "fisioterapia", doutores e demais pesquisadores, nacionalidade brasileira e formação acadêmica/titulação "graduação". Foram encontrados 47.741 currículos de fisioterapeutas disponíveis na Plataforma Lattes. O cálculo amostral foi realizado considerando a população de 47.741 currículos, margem de erro de $0,5 \%$ e nível de confiança de $95 \%$, com resultado de 25 mil currículos que foram extraídos da plataforma de forma aleatória e em seguida, planilhados e analisados.

Após a análise preliminar, foram identificados e excluídos 7.136 currículos que apresentavam dados incompletos. Dessa forma, um total de 17.864 currículos foram analisados no estudo e correspondem a aproximadamente $7,44 \%$ dos fisioterapeutas do país. É importante destacar que este recorte atende o objetivo do presente estudo que está pautado na análise dos pesquisadores fisioterapeutas, e não no perfil do fisioterapeuta como um todo.

Os currículos foram analisados por avaliadores que já apresentavam familiaridade no preenchimento e na leitura de informações na plataforma Lattes e que receberam orientações para a análise e extração dos dados. As seguintes informações foram extraídas para a composição do banco de dados: sexo, localização (considerando a região de formação ou atuação do fisioterapeuta), data da última atualização do currículo, tipo de instituição de graduação, tempo de graduação, tempo de formação (contabilizado entre o ano de sua formação até 2018), outras graduações, instituição de atuação principal, número de instituições em que atua, titulações (pós-graduação lato sensu, mestrado, doutorado e pós-doutorado), área de pós-graduação lato sensu concluída, informação sobre bolsa produtividade do $\mathrm{CNPq}$, cursos complementares, artigos publicados em periódicos, resumos publicados em anais de eventos, livros ou capítulos de livros, trabalhos apresentados em eventos, número de produções técnicas e participação e organização de eventos.

Quanto aos aspectos éticos, esta pesquisa não necessitou de aprovação ética por não haver contato dos pesquisadores com os profissionais. Além disso, os dados de identificação (nome, endereço etc.) dos profissionais não foram objeto de divulgação e mantidos sob sigilo pela equipe de pesquisadores.

Os dados coletados foram organizados em um banco de dados para análise estatística no programa Statistical Package for Social Sciences - SPSS (versão 23.0). Para as variáveis numéricas, foram realizados os cálculos de média, desvio-padrão, valor mínimo e valor máximo, e, nas categóricas, frequência e porcentagem. $\mathrm{Na}$ análise inferencial, foi realizado o teste de normalidade de Kolmogorov Smirnov, e o teste t de Student para amostras independentes, comparando sexo com o número de produções e eventos, e instituição com produções e eventos. Foi realizado o teste exato de Fisher para comparar sexo e titulações e o tipo de instituição com as titulações. A comparação entre a instituição de formação e o sexo foi realizada pelo teste Qui-quadrado, considerando o nível de significância estatística $p \leq 0,05$.

\section{RESULTADOS}

Após a organização do banco de dados dos $25 \mathrm{mil}$ currículos, foram excluídos 2.397 currículos que apresentavam deficits de informação e/ou estavam duplicados devido a atualizações da plataforma. Assim, 22.603 currículos foram tabulados e 17.864 deles, todos atualizados nos últimos 5 anos, considerados para análise estatística. Da amostra, $13.479(75,5 \%)$ eram do sexo feminino e 4.385 (24,5\%) do sexo masculino. A quantidade e distribuição dos fisioterapeutas pesquisadores pelas regiões do Brasil está informada na Tabela 1 e na Figura 1, e as características descritivas estão expressas na Tabela 2.

Tabela 1. Quantidade de fisioterapeutas pesquisadores no Brasil

\begin{tabular}{lr|}
\hline Regiões do Brasil & Frequência (\%) \\
Norte & $1005(5,62)$ \\
Nordeste & $5571(31,18)$ \\
Centro-Oeste & $1203(6,73)$ \\
Sudeste & $6259(35,04)$ \\
Sul & $2828(15,83)$ \\
Não informado & $998(5,6)$
\end{tabular}




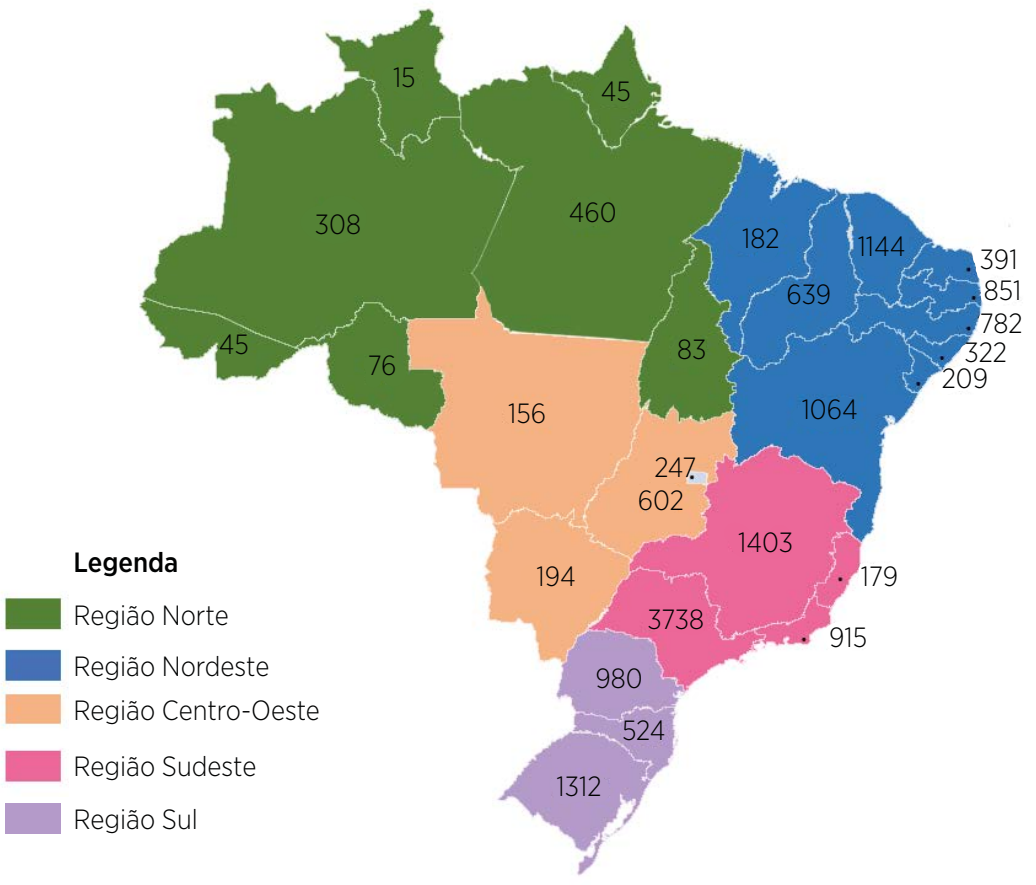

Figura 1. Distribuição dos fisioterapeutas pesquisadores pelo Brasil por estados e regiões Fonte: Elaboração própria.

Tabela 2. Características descritivas dos fisioterapeutas pesquisadores do Brasil

\begin{tabular}{|c|c|c|}
\hline Variáveis & Frequência & $\%$ \\
\hline \multicolumn{3}{|l|}{ Instituição de formação } \\
\hline Privada & 13348 & 74,7 \\
\hline Pública & 4516 & 25,3 \\
\hline \multicolumn{3}{|l|}{ Outras Graduações } \\
\hline Sim & 1186 & 6,6 \\
\hline Não & 16678 & 93,4 \\
\hline \multicolumn{3}{|l|}{ Local de Atuação Principal } \\
\hline Não informado & 5233 & 29,3 \\
\hline Hospital & 2495 & 14,0 \\
\hline Clínica & 2009 & 11,2 \\
\hline Clube Esportivo & 58 & 0,3 \\
\hline Universidade & 5301 & 29,7 \\
\hline Home Care & 207 & 1,2 \\
\hline Academia/Studio & 281 & 1,6 \\
\hline Posto de Saúde/ PSF & 138 & 0,8 \\
\hline Empresas & 240 & 1,3 \\
\hline Outros & 1902 & 10,6 \\
\hline \multicolumn{3}{|l|}{ Áreas de Pós-graduação lato sensu } \\
\hline Não realizou & 6889 & 38,60 \\
\hline Outras áreas (não reconhecidas pelo COFFITO) & 3619 & 20,30 \\
\hline Terapia Intensiva & 1235 & 6,90 \\
\hline Traumato-Ortopedia & 1226 & 6,90 \\
\hline Respiratória & 1135 & 6,40 \\
\hline Neurofuncional & 834 & 4,70 \\
\hline Terapias Manuais & 677 & 3,80 \\
\hline Dermato-Funcional & 572 & 3,20 \\
\hline Saúde da Mulher & 329 & 1,80 \\
\hline Gerontologia & 322 & 1,80 \\
\hline Cardiovascular & 257 & 1,40 \\
\hline
\end{tabular}


Tabela 2. Continuação

\begin{tabular}{lrr}
\multicolumn{1}{c}{ Variáveis } & Frequência & \% \\
Esportiva & 236 & 1,30 \\
Fisioterapia do Trabalho & 203 & 1,10 \\
Oncologia & 125 & 0,70 \\
Não informou a área de pós-graduação & 74 & 0,70 \\
Aquática & Média (DP) & Mínimo e máximo \\
& $4,19(0,93)$ & $2-10$ \\
Tempo de Graduação & $10,00(7,85)$ & $0-50$ \\
Tempo de Formado & $1,43(1,65)$ & $0-12$ \\
Número de locais de trabalho que atua & $7,14(14,58)$ & $0-541$ \\
Trabalhos apresentados em eventos & $10,72(11,53)$ & $0-181$ \\
Cursos Complementares & $2,26(8,02)$ & $0-301$ \\
Artigos Publicados & $5,03(18,96)$ & $0-610$ \\
Resumos Publicados & $0,49(2,27)$ & $0-70$ \\
Livros ou capítulos de livros & $3,09(10,63)$ & $0-423$ \\
Produções técnicas & $17,20(21,29)$ & $0-406$ \\
Participação em eventos & $2,18(4,89)$ & $0-181$
\end{tabular}

Fonte: Elaboração própria.

Os resultados referentes a formações complementares e recebimento de bolsa-produtividade pelo CNPq estão expressos nos Gráficos 1-5. Das bolsas de produtividade em pesquisa, 68 são exclusivamente da área de fisioterapia e terapia ocupacional. A análise comparativa entre sexo, tipo de instituição, produções e eventos está expressa na Tabela 3, e a análise comparativa entre sexo e tipo de instituição com a titulação está expressa na Tabela 4 .

$100 \%$

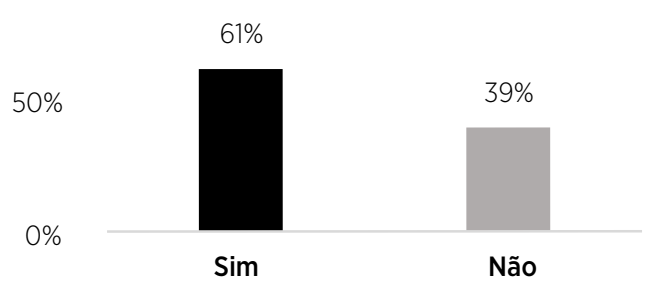

Gráfico 1. Formação complementar dos fisioterapeutas pesquisadores do Brasil: pós-graduação lato sensu

Fonte: Elaboração própria.

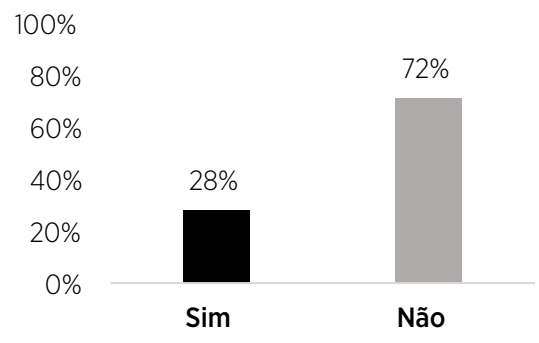

Gráfico 2. Formação complementar dos fisioterapeutas pesquisadores do Brasil: mestrado

Fonte: Elaboração própria.

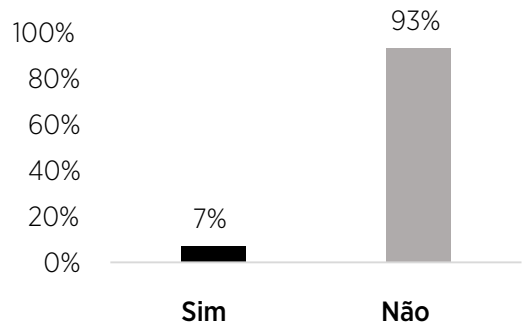

Gráfico 3. Formação complementar dos fisioterapeutas pesquisadores do Brasil: doutorado

Fonte: Elaboração própria.

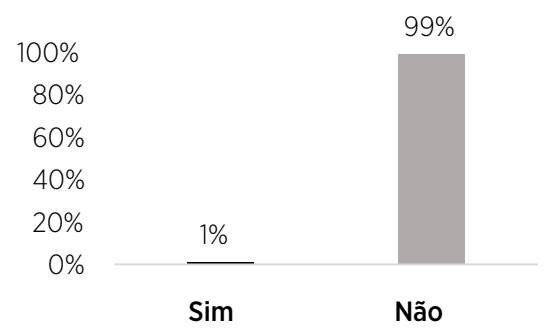

Gráfico 4. Formação complementar dos fisioterapeutas pesquisadores do Brasil: pós-doutorado

Fonte: Elaboração própria.

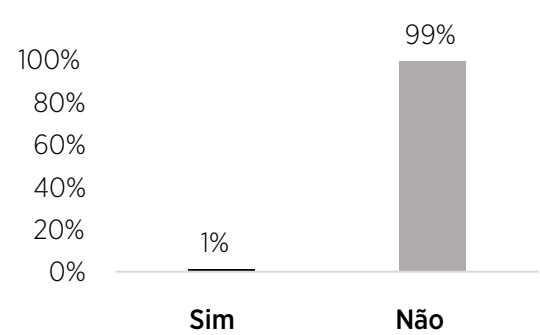

Gráfico 5. Formação complementar dos fisioterapeutas pesquisadores do Brasil: bolsista do CNPq

Fonte: Elaboração própria. 
Tabela 3. Resultados da análise comparativa entre sexo, tipo de instituição, produções e eventos dos profissionais pesquisados

\begin{tabular}{|c|c|c|c|c|c|c|c|c|c|c|}
\hline \multirow{2}{*}{$\begin{array}{c}\text { Formação } \\
\text { Complementar }\end{array}$} & \multicolumn{2}{|c|}{$\begin{array}{l}\text { Feminino } \\
(n=13479)\end{array}$} & \multicolumn{2}{|c|}{$\begin{array}{l}\text { Masculino } \\
(n=4385)\end{array}$} & \multirow[b]{2}{*}{ p } & \multicolumn{2}{|c|}{$\begin{array}{l}\text { Pública } \\
(\mathrm{n}=13348)\end{array}$} & \multicolumn{2}{|c|}{$\begin{array}{l}\text { Privada } \\
(n=4516)\end{array}$} & \multirow[b]{2}{*}{ p } \\
\hline & Média & DP & Média & DP & & Média & DP & Média & DP & \\
\hline Cursos & 10,51 & 11,11 & 11,35 & 12,70 & $<0,001^{*}$ & 11,39 & 11,78 & 10,49 & 11,43 & $<0,001^{*}$ \\
\hline $\begin{array}{l}\text { Trabalhos } \\
\text { Apresentados }\end{array}$ & 6,88 & 14,20 & 7,92 & 15,63 & $<0,001^{*}$ & 9,83 & 18,60 & 6,22 & 12,80 & $<0,001^{*}$ \\
\hline Artigos publicados & 1,97 & 6,70 & 3,13 & 11,08 & $<0,001^{*}$ & 3,17 & 9,10 & 1,95 & 7,59 & $<0,001^{*}$ \\
\hline Resumos publicados & 4,77 & 18,56 & 5,83 & 20,10 & $0,001^{*}$ & 7,55 & 20,41 & 4,18 & 18,36 & $<0,001^{*}$ \\
\hline Livros e capítulos & 0,48 & 2,26 & 0,53 & 2,28 & 0,20 & 0,65 & 2,33 & 0,44 & 2,24 & $<0,001^{*}$ \\
\hline Produções técnicas & 2,72 & 9,02 & 4,25 & 14,43 & $<0,001^{*}$ & 3,63 & 11,42 & 2,91 & 10,34 & $<0,001^{*}$ \\
\hline $\begin{array}{l}\text { Participação em } \\
\text { eventos }\end{array}$ & 16,68 & 20,11 & 18,79 & 24,50 & $<0,001^{*}$ & 19,74 & 21,74 & 16,34 & 21,07 & $<0,001^{*}$ \\
\hline $\begin{array}{l}\text { Organização } \\
\text { de eventos }\end{array}$ & 2,07 & 4,78 & 2,54 & 5,19 & $<0,001^{*}$ & 2,81 & 6,02 & 1,97 & 4,43 & $<0,001^{*}$ \\
\hline
\end{tabular}

Legenda: Foi realizada a comparação de sexo ou instituição com produções e eventos pelo teste t de Student para amostras independentes, considerando o nível de p $\leq 0,05$. Os dados foram expressos em média, desvio-padrão (DP), frequência (f) e porcentagem (\%).

Fonte: Elaboração própria.

Tabela 4. Resultados da análise comparativa entre sexo e tipo de instituição com a titulação dos profissionais pesquisados

\begin{tabular}{|c|c|c|c|c|c|c|c|c|c|c|}
\hline \multirow[b]{2}{*}{ Qualificação } & \multicolumn{2}{|c|}{ Feminino } & \multicolumn{2}{|c|}{ Masculino } & \multirow[b]{2}{*}{ p } & \multicolumn{2}{|c|}{ Pública } & \multicolumn{2}{|c|}{ Privada } & \multirow[b]{2}{*}{ p } \\
\hline & $\begin{array}{l}\operatorname{Sim} \\
f(\%)\end{array}$ & $\begin{array}{l}\text { Não } \\
\text { f (\%) }\end{array}$ & $\begin{array}{c}\text { Sim } \\
f(\%)\end{array}$ & $\begin{array}{c}\text { Não } \\
\text { f (\%) }\end{array}$ & & $\begin{array}{c}\text { Sim } \\
f(\%)\end{array}$ & $\begin{array}{l}\text { Não } \\
\text { f (\%) }\end{array}$ & $\begin{array}{l}\operatorname{Sim} \\
f(\%)\end{array}$ & $\begin{array}{l}\text { Não } \\
\text { f (\%) }\end{array}$ & \\
\hline $\begin{array}{l}\text { Pós-graduação } \\
\text { Lato Sensu }\end{array}$ & $\begin{array}{l}8079 \\
(9,9)\end{array}$ & $\begin{array}{l}5400 \\
(40,1)\end{array}$ & $\begin{array}{r}2896 \\
(66,0)\end{array}$ & $\begin{array}{r}1489 \\
(34,0)\end{array}$ & $<0,001^{*}$ & $\begin{array}{r}2720 \\
(60,2)\end{array}$ & $\begin{array}{r}1796 \\
(39,8)\end{array}$ & $\begin{array}{r}8255 \\
(61,8)\end{array}$ & $\begin{array}{r}5093 \\
(38,2)\end{array}$ & $0,05^{*}$ \\
\hline Mestrado & $\begin{array}{r}3579 \\
(26,6)\end{array}$ & $\begin{array}{r}9900 \\
(73,4)\end{array}$ & $\begin{array}{r}1408 \\
(32,1)\end{array}$ & $\begin{array}{r}2977 \\
(67,9)\end{array}$ & $<0,001^{*}$ & $\begin{array}{r}1597 \\
(35,4)\end{array}$ & $\begin{array}{r}2919 \\
(64,6)\end{array}$ & $\begin{array}{r}3390 \\
(25,4)\end{array}$ & $\begin{array}{r}9958 \\
(74,6)\end{array}$ & $<0,001^{*}$ \\
\hline Doutorado & $\begin{array}{r}976 \\
(7,2)\end{array}$ & $\begin{array}{l}12505 \\
(92,8)\end{array}$ & $\begin{array}{r}364 \\
(8,3)\end{array}$ & $\begin{array}{r}4021 \\
(91,7)\end{array}$ & $0,02^{*}$ & $\begin{array}{r}495 \\
(11,0)\end{array}$ & $\begin{array}{r}4021 \\
(89,0)\end{array}$ & $\begin{array}{r}845 \\
(6,3)\end{array}$ & $\begin{array}{l}12503 \\
(93,7)\end{array}$ & $<0,001^{*}$ \\
\hline Pós-doutorado & $\begin{array}{r}167 \\
(1,2)\end{array}$ & $\begin{array}{r}13312 \\
(98,8)\end{array}$ & $\begin{array}{r}71 \\
(1,6)\end{array}$ & $\begin{array}{r}4314 \\
(98,4)\end{array}$ & $0,05^{*}$ & $\begin{array}{r}95 \\
(2,1)\end{array}$ & $\begin{array}{r}4421 \\
(97,9)\end{array}$ & $\begin{array}{l}143 \\
(1,1)\end{array}$ & $\begin{array}{l}13205 \\
(98,9)\end{array}$ & $<0,001^{*}$ \\
\hline
\end{tabular}

Legenda: Foi realizada a comparação entre sexo ou instituição com a titulação do fisioterapeuta pesquisador foi realizada pelo teste exato de Fisher, considerando o nível de p $\leq 0,05$. Os dados foram expressos em média, desvio-padrão (DP), frequência (f) e porcentagem (\%).

Fonte: Elaboração própria.

As Tabelas 3 e 4 apontam que o sexo masculino apresenta maior número de cursos, produções cientificas, trabalhos apresentados, artigos e resumos publicados e participação e organização de eventos $(p<0,001)$. Apenas para a publicação de livros e capítulos de livros não houve diferença estatística significativa $(p=0,20)$. Em relação à formação complementar, também há predominância do sexo masculino na realização de pós-graduação lato sensu $(\mathrm{p}<0,001)$, mestrado $(\mathrm{p}<0,001)$, doutorado $(\mathrm{p}=0,02)$ e pós-doutorado $(\mathrm{p}=0,05)$.

$\mathrm{Na}$ comparação entre os tipos de instituição de ensino, verifica-se que o fisioterapeuta formado por instituição pública desenvolve mais produções e participa de mais eventos, apresentando diferença estatística significativa $(\mathrm{p}<0,001)$ em todas as variáveis analisadas - curso, trabalhos apresentados, artigos e resumos publicados, livros e capítulos, produções técnicas, participação e organização de eventos. Para a formação complementar, a pós-graduação lato sensu é mais realizada pelos fisioterapeutas formados em instituição privada $(p=0,05)$; já o mestrado, o doutorado e o pós-doutorado são mais realizados por fisioterapeutas formados em instituições públicas $(\mathrm{p}<0,001)$.

A comparação entre o tipo de instituição de ensino e o sexo foi realizada pelo teste Qui-quadrado. A análise comparativa mostrou diferença estatística significativa $(p<0,001)$, sendo a presença do sexo feminino na instituição pública de 3509 (26\%) e na instituição privada de 9970 (74\%); o sexo masculino, por sua vez, apresentou presença de 1007 (23\%) na instituição pública e de 3378 (77\%) na instituição privada.

\section{DISCUSSÃO}

O presente estudo fez um levantamento do perfil de formação e produção científica do fisioterapeuta pesquisador brasileiro a partir da base de dados de currículos pública do CNPq. Nos últimos 13 anos houve aumento exponencial na quantidade de cursos 
de fisioterapia oferecidos no Brasil, sendo São Paulo $(28,18 \%)$ e Minas Gerais (12,36\%) os estados com maior quantidade de instituições ofertando o curso de graduação 22 . $\mathrm{O}$ crescimento acelerado desses cursos, todavia, aumenta a desigualdade na formação de fisioterapeutas nas regiões do país, visto que há variações na matriz curricular e no tempo de conclusão do curso ${ }^{23}$.

Nos estados de São Paulo e do Paraná, a maioria dos fisioterapeutas tem formação em instituições privadas $84,5 \%$ e $82,2 \%$ dos profissionais, respectivamente ${ }^{3}$. Um estudo descritivo realizado com 550 instituições de ensino superior do Brasil verificou a prevalência de ofertas de vagas em instituições privadas em todas as regiões do país, representando $87,7 \%$ do total analisado ${ }^{22}$. De acordo com os dados obtidos nesse estudo, $74,7 \%$ dos fisioterapeutas são formados em instituições privadas, corroborando os estudos citados.

A prevalência da graduação privada em fisioterapia no Brasil é reflexo de uma política de expansão do ensino superior, que promoveu incentivo e liberação do ensino privado sem existir ampliação da rede pública. Assim, é esperado que a maioria dos profissionais sejam egressos do sistema privado de ensino ${ }^{17}$, dado que há uma oferta desproporcional de vagas entre as instituições de ensino do país: apenas 4.043 são em universidade públicas, o que corresponde a $5,86 \%$ do total de vagas disponíveis por ano ${ }^{8}$.

$\mathrm{Em}$ relação à formação complementar dos fisioterapeutas brasileiros, os resultados deste estudo vão de encontro com a análise documental de Cruz et al. ${ }^{23}$, sendo na pós-graduação lato sensu predominantes as áreas de neurofuncional, respiratória e de traumato-ortopedia. Porém, no presente estudo, uma porcentagem considerável (20,3\%) realizou pós-graduação lato sensu em outras áreas que não são reconhecidas pelo Conselho Federal de Fisioterapia e Terapia Ocupacional (Coffito) como saúde pública, administração em saúde, marketing em saúde, ensino e docência, entre outras. Vale ressaltar que, segundo dados do Coffito, algumas especialidades foram reconhecidas recentemente e existem áreas que apresentam o reconhecimento mais antigo, como, por exemplo, a Neurofuncional e a Pneumofuncional, reconhecidas desde 1998, e a Traumato-Ortopedia, reconhecida desde $2004^{24}$.

A região Sudeste recebe maior atenção em relação aos investimentos em pesquisa, formando profissionais mais dedicados à pesquisa ${ }^{23}$. Um estudo realizado por Treviso et al. ${ }^{25}$ demonstra que os profissionais docentes de fisioterapia apresentam maior quantidade de formação complementar - principalmente em relação à pós-graduação lato sensu.
Apesar do crescimento evidente nos últimos dez anos, a procura por programas de mestrado e doutorado ainda é pequena. Um estudo realizado com os profissionais cadastrados no Conselho Regional de Fisioterapia e Terapia Ocupacional da Oitava Região (Crefito-8) mostrou que apenas $47(12,46 \%)$ fisioterapeutas realizaram mestrado e $14(3,7 \%)$ doutorado, conformando um dos grandes desafios da profissão: a formação de novos pesquisadores, o avanço e o desenvolvimento do conhecimento ${ }^{6}$. Nesse contexto, o presente estudo constata que apenas $28 \%$ dos profissionais realizaram mestrado, e, 7\%, doutorado.

Este estudo mostra que os profissionais de fisioterapia no Brasil pesquisados atuam em universidades (29,7\%), hospitais (14\%), clínicas $(11,2 \%)$ e home care $(1,2 \%)$. Nos dados, porém, 29,3\% não informam o local de trabalho, mostrando que os pesquisadores fisioterapeutas não possuem o hábito de informar todos os locais de atuação profissional, visto que o preenchimento e a atualização da plataforma Lattes está mais relacionada a profissionais atuantes em projetos de pesquisa e vinculados às universidades pelo Brasil. Por essa perspectiva, uma pesquisa transversal analisou uma amostra de 2.323 fisioterapeutas cadastrados no Crefito-3 no estado de São Paulo e verificou que apenas $8,3 \%$ atuam em universidades; 20,1\% em hospitais; 22,6\% em clínicas e 35,4\% em home care $^{3}$. Ao comparar esses dados, é possível notar que a predominância da área de atuação para os fisioterapeutas pesquisadores ocorre em universidades; no estado de São Paulo, os fisioterapeutas atuam em home care, sendo que essa variável deve ser considerada para diferenciar o perfil do fisioterapeuta nacional e regional com o perfil do fisioterapeuta pesquisador.

No Brasil, a feminização do mercado de trabalho é decorrente do processo de modernização e mudanças culturais, fazendo com que, atualmente, $70 \%$ dos profissionais da área da saúde sejam do sexo feminino ${ }^{26}$. Uma pesquisa realizada em São Paulo mostra predominância de $80 \%$ das mulheres atuando como fisioterapeutas no estado ${ }^{3}$, e o mesmo ocorre com os egressos de fisioterapia da Universidade Federal de Minas Gerais (UFMG), em que 75\% são do sexo feminino ${ }^{27}$. Os resultados encontrados estão alinhados com essas afirmações, atestando que 75,5\% dos fisioterapeutas da amostra são mulheres.

Analisando o aspecto internacional, um estudo realizado no Nepal utilizou um questionário com o objetivo de identificar os desafios e as estratégias da formação em fisioterapia. $\mathrm{O}$ estudo identificou que a falta de incentivo e de conscientização a respeito do desenvolvimento desse 
profissional é um problema para que ele continue a busca pelo aprendizado sobre a eficácia de técnicas na área ${ }^{28}$.Um estudo realizado por Ahuja ${ }^{29}$, no Reino Unido, apontou a importância do profissional continuar se especializando e se atualizando depois de graduado. Percebe-se que há uma dificuldade do profissional fisioterapeuta em manter-se inteirado tanto em âmbito nacional como internacional.

Um estudo de revisão sistemática realizado por Silva et al. ${ }^{15}$ analisou 12 estudos a respeito do que os fisioterapeutas sabem sobre a prática baseada em evidências (PBE) e as dificuldades de aplicá-la na prática clínica. A maior parte dos estudos mostrou que os fisioterapeutas têm uma opinião favorável sobre o assunto, consideram que precisam melhorar seus conhecimento e habilidades em PBE e enfrentam algumas barreiras que dificultam a implementação na prática, tais como: falta de tempo, incapacidade de entender estatísticas, falta de apoio do empregador, falta de recursos, falta de interesse e falta de generalização dos resultados. No presente estudo, verifica-se que um dos caminhos que os fisioterapeutas têm procurado para melhorar sua formação em pesquisa são os cursos pósgraduação stricto sensu e cursos de formação complementar.

Os resultados e as interpretações deste estudo encontram limitações no que se refere à natureza da pesquisa bibliométrica. A Plataforma Lattes pode ser atualizada pelo profissional a qualquer momento e não há garantia de que as informações do currículo tenham sido publicadas na mesma época da coleta de dados. Outro ponto a ser mencionado é o preenchimento incompleto ou inadequado de informações sobre a produção técnica e bibliográfica e a ausência de informações quanto a formações complementares e atuação nos currículos dos profissionais.

Apesar das limitações, foi possível fazer um levantamento do perfil dos profissionais cadastrados e identificar o panorama dos fisioterapeutas pesquisadores atuantes no país. Os achados podem subsidiar medidas de investimentos em cursos de qualificação, formação complementar e direcionamento de profissionais para atuação em áreas deficitárias nas diferentes regiões do Brasil. Além disso, o estudo também aponta para a necessidade do preenchimento correto do currículo e que os profissionais mantenham suas informações atualizadas. Existem tutoriais na internet ensinando passo a passo como lançar as informações e está disponível gratuitamente um curso promovido pela nossa equipe de como preencher adequadamente o currículo Lattes.

Os achados podem subsidiar informações para a formação continuada e direcionamento de áreas de atuação dos fisioterapeutas em todo o país, uma vez que foram identificados os deficits quanto à distribuição geográfica, áreas de atuação e formação continuada - o que pode sinalizar as possibilidades existentes no mercado.

\section{CONCLUSÃO}

Conclui-se que, no Brasil, existe uma prevalência de fisioterapeutas pesquisadores do sexo feminino, concentrados nas regiões Sudeste e Nordeste. Esses são, em sua maioria, formados por instituições privadas, não realizaram outras graduações e trabalham em universidades. Ao analisar a formação complementar, a maioria dos profissionais realizou pós-graduação lato sensu, principalmente nas áreas de terapia intensiva e traumato-ortopedia; a minoria realizou pós-graduação stricto sensu. Os fisioterapeutas do sexo masculino apresentaram maior procura por cursos de formação complementar e os profissionais formados em instituições públicas possuem maiores indicadores de produção de trabalhos científicos, participam de mais eventos e dedicam-se a pós-graduação stricto sensu.

\section{CONTRIBUIÇÃO DOS AUTORES}

Os autores listados a seguir foram responsáveis pelo delineamento do estudo, análise e interpretação dos dados, correção da redação final e aprovação da versão final do manuscrito: Tânia Cristina Dias da Silva Hamu, Cibelle Kayenne Martins Roberto Formiga.

Os autores listados a seguir foram responsáveis pela coleta, análise, revisão da literatura e redação da versão preliminar do artigo, correção da versão final: Natália Guimarães Melo, Isabela Alves Cunha, Júlia Ferreira Alves, Amanda Lindolpho Santos, Anna Paula Nogueira, Beatriz Correa Lima, Bruna Viani Dias, Jeovana Souza Cardoso, Jhennyfer Gonzaga de Oliveira Rocha, Nathálya Pereira Portugal, Rayssa Gabrielly de Araujo, Rayssa Martins de Souza, Roberta Larissa Oliveira Paulino.

\section{AGRADECIMENTOS}

Este artigo é produto do trabalho desenvolvido pelo grupo PET (Programa de Educação Tutorial) do curso de fisioterapia da Universidade Estadual de Goiás (UEG). Instagram: @ petfisioueg. 


\section{REFERÊNCIAS}

1. Espíndola DS, Borenstein MS. Evolução histórica da fisioterapia: da massagem ao reconhecimento profissional (1894-2010). Fisioter Bras. 2011;12(5):389-94. doi: 10.33233/fb.v12i5.944

2. Soares PA. Controvérsias, dilemas e desafios profissionais dos fisioterapeutas portugueses (I). Desenvolvimento e Sociedade. 2017;(3):87-110.

3. Shiwa SR, Schmitt ACB, João SMA. O fisioterapeuta do estado de São Paulo. Fisioter Pesqui. 2016;23(3):301-10. doi: 10.1590/1809-2950/16115523032016

4. Silva DCP, Grazziano CR, Carrascosa AC. Satisfação profissional e perfil de egressos em fisioterapia. ConScientiae Saúde. 2018;17(1):65-71. doi: 10.5585/conssaude.v17n1.7694

5. Brasil. Decreto-Lei no 938, de 13 de outubro de 1969. Provê sobre as profissões de fisioterapeuta e terapeuta ocupacional, e dá outras providências. Diário Oficial da União [Internet]. 1969 Oct 13 [cited 2020 June 11]. Available from: https://bit.ly/2RpGSzN

6. Mariotti MC, Bernardelli RS, Nickel R, Zeghbi AA, Teixeira MLV, Costa Filho RM. Características profissionais, de formação e distribuição geográfica dos fisioterapeutas do Paraná - Brasil. Fisioter Pesqui. 2017;24(3):295-302. doi: 10.1590/1809-2950/16875724032017

7. Landry MD, Hack LM, Coulson E, Freburguer J, Johnson MP, Katz R, et al. Workforce Projections 2010-2020: Annual Supply and Demand Forecasting Models for Physical Therapists Across the United States. Phys Ther. 2016;96(1):71-80. doi: 10.2522/ ptj.20150010

8. Koetz LCE, Périco E, Grave MQ. Distribuição geográfica da formação em fisioterapia no Brasil: crescimento desordenado e desigualdade regional. Trab Educ Saúde. 2017;15(3):917-30. doi: 10.1590/1981-7746-sol00070

9. Coury HJCG, Vilella, I. Perfil do pesquisador fisioterapeuta brasileiro. Braz J Phys Ther. 2009;13(4):356-63. doi: 10.1590/ S1413-35552009005000048

10. Ferigollo JP, Fedosse E, Santos Filha VAV. Qualidade de vida de profissionais da saúde pública. Cad Ter Ocup. 2016;24(3):497507. doi: 10.1590/1413-81232018234.09292016

11. Shannon G, Minckas N, Tan D, Haghparast-Bidgoli H, Batura N, Mannell J. Feminisation of the health workforce and wage conditions of health professions: an exploratory analysis. Hum Resour Health. 2019;17(1):72-84. doi: 10.1186/s12960-019-0406-0

12. Gonçalves RF, Sandes AAG, Nascimento IYM, Amaral ARM, Araújo RC, Silva TFA. Avaliação dos cursos de fisioterapia nos anos de 2004 a 2013. Fisioter Pesqui. 2017; 24(4):392-98. doi: 10.1590/1809-2950/17167124042017

13. Instituto Brasileiro de Geografia e Estatística. Estimativas da população residente para os municípios e para as unidades da federação com data de referência em 1ำ de julho de 2018 [Internet]. Rio de Janeiro: IBGE; 2018 [cited 2020 Jul 21]. Available from: https://bit.ly/2Q28tXy

14. Conselho Federal de Fisioterapia e Terapia Ocupacional. COFFITO defende que quiropraxia é uma especialidade da fisioterapia. Brasília: COFFITO; 2018. [cited 2020 Jun 20]. Available from: https://bit.ly/32foA6M

15. Silva TM, Costa LCM, Garcia AN, Costa LOP. What do physical therapists think about evidence-based practice? A systematic review. Man Ther. 2015;20(3):388-401. doi: 10.1016/j. math.2014.10.009

16. Kamper, SJ. Evidence in practice: a new series for clinicians. J Orthop Sports Phys Ther. 2018;48(6):429. doi: 10.2519/ jospt.2018.0105

17. Bispo Júnior JP. Formação em fisioterapia no Brasil: reflexões sobre a expansão do ensino e os modelos de formação. Hist Cienc Saude Manguinhos. 2009;16(3):655-68. doi: 10.1590/ S0104-59702009000300005

18. Costa LOP, Moseley AM, Sherrington C, Maher CG, Herbert RD, Elkins MR. Core journals that publish clinical trials of physical therapy interventions. Phys Ther. 2010;90(11):1631-40. doi: 10.2522/ptj.20090419

19. Habibi SSS, Dehkordi SN, Dadgoo M, Lajevardi L. Physiotherapists' perception of evidence-based practice. J Mod Rehabil. 2016;10(4):169-76. doi: 10.18869/nirp.jmr.10.4.169

20. Seelro U, Khan MS, Tanveer E, Khan M, Kumar V, Ahmed N. Knowledge and Attitude towards Evidence Based Practice among the Physiotherapists. IJCRR. 2018;9(9):20602-9. doi: 10.15520/ijcrr/2018/9/09/598

21. Calvalcante CCL, Rodrigues ARS, Dadalto TV, Silva EB. Evolução científica da fisioterapia em 40 anos de profissão. Fisioter Mov. 2011;24(3):513-22. doi: 10.1590/S0103-51502011000300016

22. Góes AB, Araújo FRO, Marques AP, Schmitt ACB. Overview of physical therapy graduation courses in Brazil: current scenario. Fisioter Mov. 2017;30(4):661-9. doi: 10.1590/19805918.030.004.ao01

23. Cruz FG, Cedro TAN, Camargo SB, Sá KN. Scientometric profile of physiotherapists Brazilian scientists. Fisioter Mov. 2018;31(1):e003123. doi: 10.1590/1980-5918.031.ao23

24. Conselho Nacional de Fisioterapia e Terapia Ocupacional. Especialidades reconhecidas pelo COFFITO [Internet]. Brasília: COFFITO; 2014 [cited 2020 Jul 6]. Available from: https://bit. ly/3mMwptM

25. Treviso P, Costa BEP. Percepção de profissionais da área da saúde sobre a formação em sua atividade docente. Texto Contexto Enferm. 2017;26(1):e5020015. doi: 10.1590/0104-07072017005020015

26. Matos IB, Toassi RFC, Oliveira MC. Profissões e ocupações de saúde e o processo de feminização: tendências e implicações. Athenea Digital. 2013;13(2):239-44.

27. Camara AMCS, Santos LLCP. Um estudo com egressos do curso de fisioterapia da Universidade Federal de Minas Gerais (UFMG): 1982-2005. Rev Bras Educ Med. 2012;36(1):5-17. doi: 10.1590/S0100-55022012000200002

28. Acharya RS, Adhikari SP, Oraibi SA, Baidya S. Challenges and future development of physiotherapy education in Nepal. IJCRR 2015;7(13):35-42.

29. Ahuja D. Continuing professional development within physiotherapy: a special perspective. J Phys Ther. 2011;3(1):4-8. 